\title{
The importance of early completion of cardiac investigations after ischaemic stroke: a case and systematic review of reperfusion therapy in stroke due to cardiac fibroelastoma
}

\author{
Authors: Henrietta Helliwell, ${ }^{\mathrm{A}}$ Aishwarya Desai, ${ }^{\mathrm{B}}$ Mark McCole, ${ }^{\mathrm{C}}$ Andy Beale, ${ }^{\mathrm{D}}$ Gopinath Ramadurai, ${ }^{\mathrm{E}}$ \\ Badrinathan Chandrasekaran ${ }^{F}$ and Gabriel SC Yiin ${ }^{G}$
}

\begin{abstract}
KEYWORDS: stroke, cardiac investigations, reperfusion therapy, cardiac fibroelastoma
\end{abstract}

DOI: $10.7861 /$ clinmed.2020-0763

\section{Introduction}

We describe a rare case of a 69-year-old woman presenting with sudden onset right-sided weakness, sensory disturbance and dysarthria due to a left-middle cerebral artery territory stroke. The patient was successfully thrombolysed, but shortly developed a recurrent stroke in a different vascular territory. Transthoracic echocardiography showed a $2 \times 1 \mathrm{~cm}$ mobile structure on the aortic valve. This was resected and histology identified a fibroelastoma. This case highlights the importance of early completion of cardiac investigations in stroke patients, and that a known cardiac papillary fibroelastoma (CPF) should not be considered a contraindication to thrombolysis or thrombectomy.

\section{Case presentation}

A 69-year-old woman, with a past history of migraine with aura, hypercholesterolaemia, herpes simplex keratitis and a previous right carotid artery dissection, presented to the emergency department with sudden onset of right-sided weakness, sensory disturbance and dysarthria.

Examination revealed a right hemiparesis with hemi-sensory loss and her National Institute of Health Stroke Scale (NIHSS) score

\footnotetext{
Authors: ${ }^{\text {f }}$ foundation year-2 doctor in stroke medicine, Great Western Hospitals NHS Foundation Trust, Swindon, UK; ${ }^{\text {B }}$ geriatric medicine registrar, Great Western Hospitals NHS Foundation Trust, Swindon, UK; ' Consultant histopathologist, John Radcliffe Hospital, Oxford, UK; ${ }^{D}$ consultant radiologist, Great Western Hospitals NHS Foundation Trust, Swindon, UK; ${ }^{\mathrm{E}}$ Consultant stroke physician, Gloucestershire Hospitals NHS Foundation Trust, Cheltenham, UK; ${ }^{F}$ consultant cardiologist, Great Western Hospitals NHS Foundation Trust, Swindon, UK; ${ }^{G}$ consultant neurologist, Great Western Hospitals NHS Foundation Trust, Swindon, UK
}

was 6. Systemic examination and observations were normal, and there were no additional heart murmurs.

Computed tomography (CT) of the brain showed no focal intracranial abnormalities and she subsequently underwent intravenous thrombolysis with alteplase. CT of the brain performed 24 hours post-thrombolysis identified a small focal infarction in the posterior left lentiform nucleus and external capsule. Doppler ultrasound of both carotid arteries were normal. Her blood tests showed mildly elevated cholesterol and electrocardiography (ECG) showed sinus rhythm.

She improved with rehabilitation in the stroke unit. Her NIHSS was 1 and she had a modified Rankin score of 2 upon discharge with atorvastatin, perindopril, ranitidine and clopidogrel. As part of her on-going outpatient investigations, she underwent a 72-hour Holter ECG monitoring 2 months after discharge. It identified a single episode of ventricular tachycardia that lasted for 10 beats, and prompted a request for transthoracic echocardiography (TTE). She suffered a minor posterior circulation stroke a week before the TTE, which subsequently identified a $2 \times 1$ $\mathrm{cm}$ mobile structure on the aortic valve (Figs 1 and 2).

The patient was admitted directly to the coronary care unit for further investigation. Repeat inflammatory markers and three sets of blood cultures were normal. A CT cardiac coronary angiography did not show any evidence of coronary artery disease, but confirmed that the mass was attached to the anterior leaflet of the aortic valve extending into the supravalvular aorta (Fig 1).

She was transferred to a tertiary centre for surgical resection of the mass. Subsequent histology had identified it as a fibroelastoma (Fig 3). Repeated TTE 2 years post-surgical removal showed no evidence of recurrence of the fibroelastoma with preserved left ventricular systolic function and mild aortic regurgitation.

\section{Discussion}

Our case demonstrated cerebral embolisation secondary to a CPF, a rare cause of stroke and successful treatment with reperfusion therapy. CPFs are benign tumours, accounting for $<10 \%$ of all cardiac tumours but the most common type of valvular tumour. ${ }^{1,2}$ The majority of CPFs are found at the aortic valve, with the left ventricle as the most common non-valvular site. ${ }^{3-5}$ The mean age 
Fig 1. Transthoracic echocardiography showing the fibroelastoma (white arrow) on the right coronary cusp. a) Parasternal long axis view. b) Modified four-chamber apical view. c) Normal Doppler velocity across the valve. d) Parasternal short axis view.

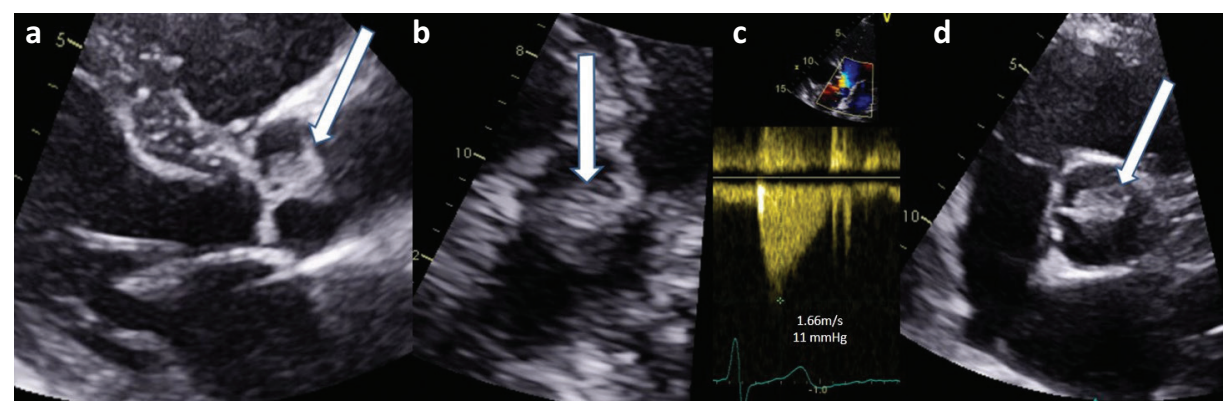

of onset was 66 years and the size ranged from $4-70 \mathrm{~mm} .{ }^{6}$ It has a sea anemone-like appearance macroscopically, and microscopic examination reveals an avascular frond formed by a fibroelastic core with spindle cells and an endocardial covering. ${ }^{5}$

CPFs are usually found incidentally, but have presented in a number of ways, including transient ischaemic attack/stroke, heart failure and even sudden death. ${ }^{5}$ In this case, the patient's ventricular tachycardia is postulated to be secondary to the CPF, perhaps due to cardiac ischaemia caused by obstruction of the blood supply to the coronary arteries. ${ }^{6}$ Conversely, right-sided tumours are often asymptomatic until sizeable enough to restrict intracardiac flow or induce arrhythmias. ${ }^{4} \mathrm{CPF}$ has a $1.6 \%$ risk of recurrence after resection, and requires regular postoperative surveillance. ${ }^{6}$

Along with this case, a detailed search of PUBMED and EMBASE revealed 11 additional cases (supplementary material S1) that had described good outcomes with patients undergoing reperfusion therapy in strokes secondary to CPFs. ${ }^{2,3,8-14}$ However, all but one had a histological examination of the retrieved clot that confirmed a fragment of the tumour, and hence one could not exclude the possibility that some of the embolised clots in these studies contained predominantly thrombus rather than the tumour material, which could in turn explain the good prognosis. ${ }^{11}$ Once identified, CPFs should be removed as soon as possible due to the risk of recurrent embolic events, while balancing the increased risk of bleeding during cardiopulmonary bypass. , $^{4,6}$ Other modifiable risk factors for stroke should also be adequately treated.

Clinicians are increasingly aware of the importance of detection of paroxysmal atrial fibrillation after transient ischaemic attack and stroke but the clinical utility of echocardiography is uncertain. ${ }^{15}$ Despite that, the Royal College of Physicians' stroke guideline has recommended TTE in patients with

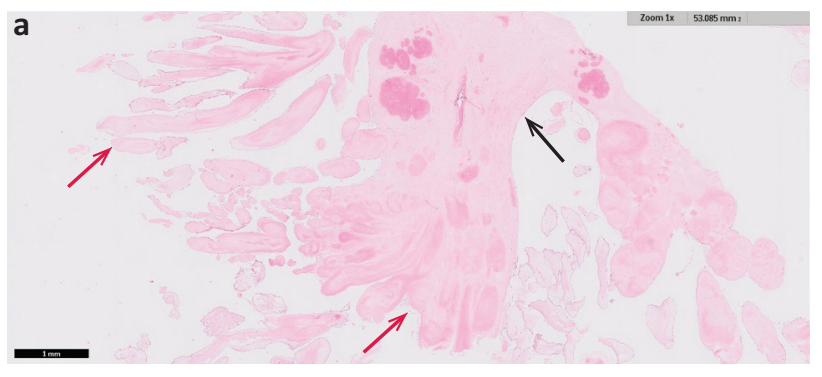

b

Fig 3. Haematoxylin and eosin stain of papillary fronds. a) Papillary fronds (red arrows) arising from a central stalk (black arrow) at low magnification. b) Papillary fronds showing the fibroelastic core covered with bland endothelium at higher magnification.

transient ischaemic attack or stroke who have clinical or electrocardiographic features of structural cardiac disease, and in those with undetermined aetiology especially if brain imaging is supportive of cardioembolism. ${ }^{16}$ One could even speculate that this patient's recurrent stroke might have been potentially preventable with faster completion of cardiac investigations.
Fig 2. Computed tomography showing fibroelastoma (white arrow) and right aortic valve cusp (red arrow). a) Maximum intensity projection of the aortic valve showing left coronary artery ostia (LCO) and right coronary artery ostia (RCO). b) Maximum intensity projection through the aortic valve plane. c) 3D volume rendering technique of the aortic valve showing the three cusps, and the attachment of the fibroelastoma to the right coronary cusp.

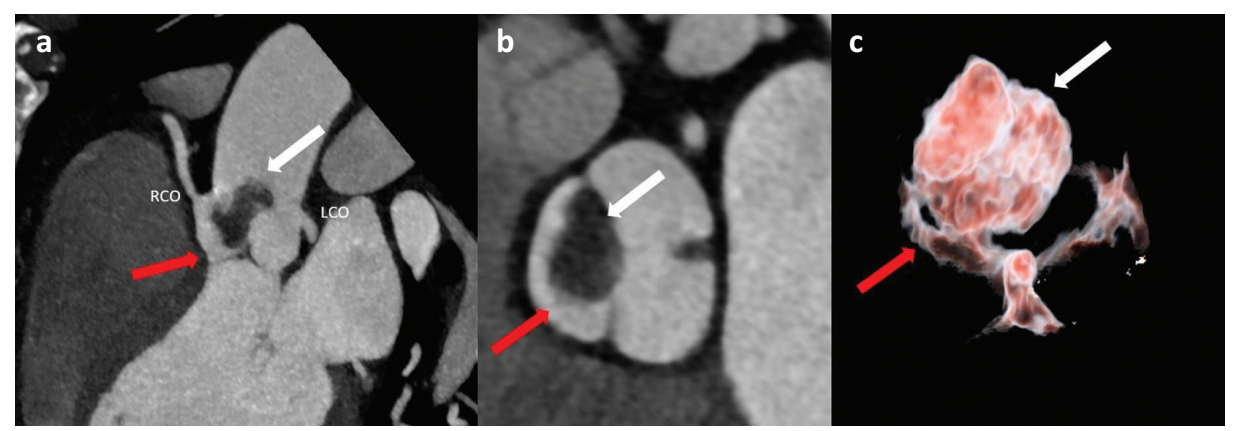




\section{Conclusion}

$\mathrm{CPF}$ is the most common cardiac tumour and has high risk of cerebral embolisation. Patients with a known CPF should not be considered a contraindication to thrombolysis or thrombectomy and tend to have a good outcome after such therapies. Cardiac investigations should be completed early in stroke patients with undetermined aetiology.

\section{Supplementary material}

Additional supplementary material may be found in the online version of this article at www.rcpjournals.org/clinmedicine: S1 - Summary of search of PUBMED and EMBASE revealing 11 additional cases that describe good outcomes with patients undergoing reperfusion therapy in strokes secondary to cardiac papillary fibroelastomas.

\section{References}

1 Vukmirović F, Vukmirović M, Tomašević-Vukmirović I. Papillary fibroelastoma of the aortic valve. Vojnosanitetski Pregled 2014; 71:600-2.

2 Kumar V, Soni P, Hashmi A, Moskovits M. Aortic valve fibroelastoma: a rare cause of stroke. BMJ Case Rep 2016;2016:bcr2016217631.

3 Grolla E, Dalla Vestra M, Zoffoli G et al. Papillary fibroelastoma, unusual cause of stroke in a young man: a case report. J Cardiothorac Surg 2017;12:33.

4 Itrat A, George P, Khawaja Z et al. Pathological evidence of cardiac papillary fibroelastoma in a retrieved intracranial embolus. Can J Neurol Sci 2015;42:66-8.

5 Gowda RM, Khan IA, Nair CK et al. Cardiac papillary fibroelastoma: a comprehensive analysis of 725 cases. Am Heart J 2003;146: 404-10.

6 Tamin SS, Maleszewski J], Scott CG et al. Prognostic and bioepidemiologic implications of papillary fibroelastomas. J Am Coll Cardiol 2015;65:2420-9.
7 Tavares CMDF, De Oliveira NA, Miguel R, Atié J. Recurrent ventricular fibrillation secondary to aortic valve tumor. Heart Rhythm 2004;1:348-51.

8 Bonavia L, Jackson J, Law JPY, Yii M, Joshi D. Brainstem stroke caused by left atrial cardiac papillary fibroelastoma: an increasingly recognised rare cause of stroke. Cardiovasc Pathol 2019:40:65-7.

9 Moreno-Estébanez A, Rodríguez AL, Serrano IU et al. Papillary fibroelastoma, an unexpected cause of stroke: Outcomes after acute reperfusion therapy. Neurol Clin Pract 2018;8:e4-6.

10 Santos A, Pinho J, Ramos V et al. Stroke and cardiac fibroelastoma: mechanical thrombectomy after thrombolytic therapy. J Stroke Cerebrovasc Dis 2014:23:1262-4.

11 Tejada J, Galiana A, Balboa Ó et al. Mechanical endovascular procedure for the treatment of acute ischemic stroke caused by total detachment of a papillary fibroelastoma. BMJ Case Rep 2013:2013:bcr2013010800.

12 Corrado G, Panisi P, Checcarelli N, Ambrosiani L. An unusual cause of ischaemic stroke with successful thrombolysis. J Stroke Cerebrovasc Dis 2013;22:e691-2.

13 Ljeval J, Mišmaš A, Bazina A et al. An infrequent type of stroke with an unusual cause and successful therapy: basilar artery occlusion caused by a cardiac papillary fibroelastoma recanalised 12 hours after onset. Intern Med 2013;52:277-9.

14 Matjević V, Poljaković Z, Ilić I, Cikeš I, Habek M. Cardiac papillary fibroelastoma: source of cerebral embolism treated with intravenous thrombolysis. J Stroke Cerebrovasc Dis 2011;20:485-7.

15 Sposato LA, Cipriano LE, Saposnik G et al. Diagnosis of atrial fibrillation after stroke and transient ischaemic attack: a systematic review and meta-analysis. Lancet Neurol 2015;14:377-87.

16 Royal College of Physicians. National clinical guideline for stroke. London: RCP, 2016. www.strokeaudit.org/SupportFiles/Documents/ Guidelines/2016-National-Clinical-Guideline-for-Stroke-5t-(1).aspx [Accessed 19 June 2020].

Address for correspondence: Dr Henrietta Helliwell, Great Western Hospital, Marlborough Road, Swindon SN3 6BB, UK. Email: h.helliwell@nhs.net 